\title{
The Future of Web Interfaces
}

\author{
Steven Pemberton \\ CWI and W3C \\ steven.pemberton@cwi.nl
}

\begin{abstract}
The Web took the world by storm, and as a result developed rapidly in many directions. However it still exhibits many aspects of its early development, such as its visual and computer-screen orientation. But the Web is still developing rapidly: there are now more browsers on mobile telephones than on desktops, and there is a vast diversity in types of devices, types and orientations of screens, and sizes (in number of pixels), and resolutions (in dpi)
\end{abstract} of screens.

Dealing with this diversity is impossible to address just by keeping a list of all the possible devices, or even a list of the most-used ones, and producing different sites for them, since the complexity would be unmanageable, and because once sites started turning away browsers and devices they didn't know, the browser makers responded by disguising themselves to such sites as other browsers.

On top of this diversity there is also the diversity required for accessibility. Although providing access for the visually impaired is an important reason for accessibility, we are all more or less visually impaired at one time or another. When displaying an application on a projector screen at a conference or meeting, the whole audience will typically be visually impaired in comparison to someone sitting behind a computer screen. The existence of separate socalled "Ten-foot Interfaces" (for people controlling their computers by remote control from an armchair ten feet away) demonstrates that the original applications are not designed for accessibility. Furthermore, Google (and all other search engines) is blind, and sees only what a blind user sees of a page; as the webmaster of a large bank has remarked, "we have noticed that improving accessibility increases our Google rating".

The success of the Web has turned the browser into a central application area for the user, and you can spend most of your day working with applications in the browser, reading mail, shopping, searching your own diskdrive. The advent of applications such as Google Maps and GMail has focussed minds on delivering applications via the web, not least because it eliminates the problems involved with versioning: everyone always has the most recent version of your application. Since Web-based applications have benefits for both user and provider, we can only expect to see more of them in the future.

But this approach comes at a cost. Google Maps is of the order of $200 \mathrm{~K}$ of Javascript code. Such applications are only writable by programming experts, and producing an application is not possible by the sort of people who often produce web pages for their own use.

The Web Interfaces landscape is in turmoil at the moment. Microsoft has announced a new markup language and vector graphics language for the next 
version of Windows; probably as a response Adobe has acquired Macromedia and therefore Flash; W3C have standards for applications in the form of XForms, XHTML and SVG and are working on 'compound documents'; and other browser manufacturers are calling for their own version of HTML.

What are we to make of these different approaches? Are they conflicting? Have any addressed authorability, device-independence, usability or accessibility? Is it even possible to make accessible applications? HTML made creating hypertext documents just about as easy as it could be; do any of the new approaches address this need for simplicity, or has power been irretrievably returned to the programmers?

This talk discusses the requirements for Web Applications, and the underpinnings necessary to make Web Applications follow in the same spirit that engendered the Web in the first place. 